\title{
MINIMAL SETS OF PERIODS FOR MORSE-SMALE DIFFEOMORPHISMS ON NON-ORIENTABLE COMPACT SURFACES WITHOUT BOUNDARY
}

\author{
JAUME LLIBRE ${ }^{1}$ AND VÍCTOR F. SIRVENT ${ }^{2}$
}

\begin{abstract}
We study the minimal set of (Lefschetz) periods of the $C^{1}$ MorseSmale diffeomorphisms on a non-orientable compact surface without boundary inside its class of homology. In fact our study extends to the $C^{1}$ diffeomorphisms on these surfaces having finitely many periodic orbits all of them hyperbolic and with the same action on the homology as the Morse-Smale diffeomorphisms.

We mainly have two kind of results. First we completely characterize the minimal sets of periods for the $C^{1}$ Morse-Smale diffeomorphisms on non-orientable compact surface without boundary of genus $g$ with $1 \leq g \leq 9$. But the proof of these results provides an algorithm for characterizing these minimal sets of periods for the $C^{1}$ Morse-Smale diffeomorphisms on non-orientable compact surfaces without boundary of arbitrary genus.

Second we study what kind of subsets of positive integers can be minimal sets of periods of the $C^{1}$ Morse-Smale diffeomorphisms on a non-orientable compact surface without boundary.
\end{abstract}

\section{INTRODUCTION}

In the study of the discrete dynamical systems and, in particular in the study of the orbits of self-maps defined on a given compact manifold, the periodic orbits plays an important role. These last forty years there was many results showing that some simple assumptions force qualitative and quantitative properties (like the set of periods) of a map. One of the first results in this direction was the paper Period three implies chaos for the interval continuous self-maps, see [19].

Probably for continuous self-maps on compact manifolds the most useful tool for studying the existence of fixed and periodic points is the Lefschetz Fixed Point Theorem and its improvements, see for instance $[1,2,6,7,8,10,15,16,20,23]$. The Lefschetz zeta function $\zeta_{f}(t)$ simplifies the study of the periodic points of $f$. It is a generating function for all the Lefschetz numbers of all iterates of $f$. All these notions are defined in section 3 .

Here we restrict our attention to the relevant class of discrete smooth dynamical systems given by the Morse-Smale diffeomorphisms. We recall some basic definitions and notation that will allow to define them.

Key words and phrases. Morse-Smale diffeomorphism, Lefschetz number, zeta function, set of periods, minimal set of periods, non-orientable compact surfaces.

2000 Mathematics Subject Classification: 37D15, 37E15.

The first author is supported in part by MCI/FEDER grant number MTM2009-03437 and by CICYT grant number 2009SGR-410. The second author would like to thank the Department of Mathematics at the Universitat Autònoma de Barcelona for its hospitality. The research of this article was partially done during a visit of the second author to this institution. 
We denote by $\operatorname{Diff}(M)$ the space of $\mathcal{C}^{1}$ diffeomorphisms on a compact manifold $M$. This space is a topological space endowed with the topology of the supremum with respect to $f$ and its differential $D f$. In this paper all the diffeomorphisms will be $\mathcal{C}^{1}$.

Two diffeomorphisms $f, g \in \operatorname{Diff}(M)$ are $C^{1}$ equivalent if and only if there exists a $C^{1}$ homeomorphism $h: M \rightarrow M$ such that $h \circ f=g \circ h$. A diffeomorphism $f$ is structurally stable provided that there exists a neighborhood $\mathcal{U}$ of $f$ in $\operatorname{Diff}(M)$ such that each $g \in \mathcal{U}$ is topologically equivalent to $f$. Since the class of MorseSmale diffeomorphisms is structurally stable inside the class of all diffeomorphisms (see $[26,27,25]$ ), to understand the dynamics of this class is an interesting problem.

Many papers has been published analyzing the relationships between the dynamics of the Morse-Smale diffeomorphisms and the topology of the manifold where they are defined, see for instance $[3,4,5,10,11,12,13,14,24,26,28,29,30]$. The Morse-Smale diffeomorphisms have a relatively simple orbit structure. In fact, their sets of periodic orbits are finite, and their structure is preserved under small $C^{1}$ perturbations.

Our main goal is to study the minimal sets of periods for the Morse-Smale diffeomorphisms on non-orientable compact surfaces without boundary. In fact our study is more general, it will work for $C^{1}$ maps on these surfaces having all the periodic points hyperbolic and with the same action on the homology as the Morse-Smale diffeomorphisms.

Let $M$ be a $C^{1}$ compact surface. Let $f: M \rightarrow M$ be a $C^{1}$ diffeomorphism. A point $x \in M$ is nonwandering of $f$ if for any neighborhood $\mathcal{U}$ of $x$ there exists some positive integer $m$ such that $f^{m}(\mathcal{U}) \cap \mathcal{U} \neq \emptyset$. The set of nonwandering points of $f$ is denoted by $\Omega(f)$.

We say that $x$ is a periodic point of $f$ of period $p$ if $f^{p}(x)=x$ and $f^{j}(x) \neq x$ for all $0 \leq j<p$. The set $\left\{x, f(x), \ldots, f^{p-1}(x)\right\}$ is called the periodic orbit of the periodic point $x$. Let $x$ be a periodic point of period $p$, it is called hyperbolic if the eigenvalues of $D f^{p}(x)$ have norm different from 1 .

If $x$ is a hyperbolic periodic point of $f$ of period $p$, the stable manifold of $x$ is

$$
W^{s}(x)=\left\{y \in M: d\left(x, f^{p m}(y)\right) \rightarrow 0 \text { as } m \rightarrow \infty\right\}
$$

and the unstable manifold of $x$ is

$$
W^{u}(x)=\left\{y \in M: d\left(x, f^{p m}(y)\right) \rightarrow 0 \text { as } m \rightarrow-\infty\right\},
$$

where $d$ is a distance on $M$ induced by the norm of the supremum.

A diffeomorphism $f: M \rightarrow M$ is Morse-Smale if

(i) $\Omega(f)$ is finite,

(ii) all periodic points are hyperbolic, and

(iii) for each $x, y \in \Omega(f), W^{s}(x)$ and $W^{u}(y)$ have transversal intersections.

The first condition implies that $\Omega(f)$ is the set of all periodic points of $f$.

Let

$$
\operatorname{Per}(f)=\{m \in \mathbb{N}: f \text { has a periodic orbit of period } m\}
$$

be the set of periods of $f$.

We recall that a non-orientable compact surface without boundary of genus $g>0$ is the connected sum of $g$ real projective planes. In the rest of this paper we shall assume that $M=N_{g}$ is a non-orientable compact surface without boundary of genus $g>0$, unless it is stated otherwise. 
The Lefschetz zeta function $\zeta_{f}(t)$ for a $C^{1}$ Morse-Smale diffeomorphism $f$ on a surface $N_{g}$ is introduced in section 3 . Using this function we define the minimal set of Lefschetz periods $\operatorname{MPer}_{L}(f)$ for a such diffeomorphism $f$ in section 4 . As we shall see the study of this set is important because any other $C^{1}$ Morse-Smale diffeomorphism $h$ on a surface $N_{g}$ in the same homology class than $f$ satisfies

$$
\operatorname{MPer}_{L}(f) \subseteq \operatorname{Per}(h) \text {. }
$$

The set $\operatorname{MPer}_{L}(f)$ is computable from the Lefschetz zeta function of $f$. In Section 4 we describe how to compute in a systematic way the Lefschetz zeta functions $\zeta_{f}(t)$ of all the $C^{1}$ Morse-Smale diffeomorphisms on $N_{g}$ for an arbitrary genus $g$.

One of our main results is the complete classification of the minimal sets of Lefschetz periods for the $C^{1}$ Morse-Smale diffeomorphisms on non-orientable compact surfaces without boundary of genus $g$ with $1 \leq g \leq 9$ stated in Theorem 8 of section 5. As we shall see the proof of this theorem provides an algorithm for characterizing the minimal sets of Lefschetz periods for the $C^{1}$ Morse-Smale diffeomorphisms on non-orientable compact surfaces without boundary of arbitrary genus.

In the rest of section 5 we study what kind of subsets of positive integers can be minimal sets of Lefschetz periods for the $C^{1}$ Morse-Smale diffeomorphisms on a nonorientable compact surface without boundary. Our main results in this direction are summarized in Theorem 9. We also state an open question at the end of section 5 .

The study of the minimal sets of Lefschetz periods for the $C^{1}$ Morse-Smale diffeomorphisms on an orientable compact surface without boundary has been made in $[3,4,5,11,12,14,21,22,24]$.

A key point for computing the Lefschetz zeta functions of the $C^{1}$ Morse-Smale diffeomorphisms on an non-orientable compact surface without boundary are the cyclotomic polynomials. In Section 2 we introduce these polynomials and some of their basic properties.

\section{Cyclotomic polynomials}

Let $n$ denote an integer. The $n$-th cyclotomic polynomial is given by

$$
c_{n}(t)=\frac{1-t^{n}}{\prod_{\substack{d \mid n \\ d<n}} c_{d}(t)},
$$

for $n>1$ and $c_{1}(t)=1-t$. An alternative way to express $c_{n}(t)$ is

$$
c_{n}(t)=\prod_{k}\left(w_{k}-t\right),
$$

for $n \neq 2$, where $w_{k}=e^{2 \pi i k / n}$ and $k$ runs over the relative primes to $n$ and smaller than $n$, for $c_{2}(t)=-\left(w_{2}-t\right)$. For more details about these polynomials see [18].

Let $\varphi(n)$ be the degree of $c_{n}(t)$. Then $n=\sum_{d \mid n} \varphi(d)$. So $\varphi(n)$ is the Euler function, which satisfies

$$
\varphi(n)=n \prod_{\substack{p \mid n \\ p \text { prime }}}\left(1-\frac{1}{p}\right) .
$$


Therefore if the prime decomposition of $n$ is $p_{1}^{\alpha_{1}} \cdots p_{k}^{\alpha_{k}}$, then

$$
\varphi(n)=\prod_{j=1}^{k} p_{j}^{\alpha_{j}-1}\left(p_{j}-1\right) .
$$

From the formula (1), we have

$$
c_{n}(t)=\prod_{d \mid n}\left(1-t^{d}\right)^{\mu(n / d)}
$$

where $\mu$ is the Möbius function, i.e.

$$
\mu(m)= \begin{cases}1 & \text { if } m=1 \\ 0 & \text { if } k^{2} \mid m \text { for some } k \in \mathbb{N} \\ (-1)^{r} & \text { if } m=p_{1} \cdots p_{r} \text { has distinct primes factors. }\end{cases}
$$

TABLE 1. The first thirty cyclotomic polynomials.

$$
\begin{aligned}
& c_{1}(t)=1-t \quad c_{2}(t)=1+t \quad c_{3}(t)=\frac{1-t^{3}}{1-t} \\
& c_{4}(t)=1+t^{2} \quad c_{5}(t)=\frac{1-t^{5}}{1-t} \quad c_{6}(t)=\frac{1+t^{3}}{1+t} \\
& c_{7}(t)=\frac{1-t^{7}}{1-t} \quad c_{8}(t)=1+t^{4} \quad c_{9}(t)=\frac{1-t^{9}}{1-t^{3}} \\
& c_{10}(t)=\frac{1+t^{5}}{1+t} \quad c_{11}(t)=\frac{1-t^{11}}{1-t} \quad c_{12}(t)=\frac{1+t^{6}}{1+t^{2}} \\
& c_{13}(t)=\frac{1-t^{13}}{1-t} \quad c_{14}(t)=\frac{1+t^{7}}{1+t} \quad c_{15}(t)=\frac{\left(1-t^{15}\right)(1-t)}{\left(1-t^{3}\right)\left(1-t^{5}\right)} \\
& c_{16}(t)=1+t^{8} \quad c_{17}(t)=\frac{1-t^{17}}{1-t} \quad c_{18}(t)=\frac{1+t^{9}}{1+t^{3}} \\
& c_{19}(t)=\frac{1-t^{19}}{1-t} \quad c_{20}(t)=\frac{1+t^{10}}{1+t^{2}} \quad c_{21}(t)=\frac{\left(1-t^{21}\right)(1-t)}{\left(1-t^{3}\right)\left(1-t^{7}\right)} \\
& c_{22}(t)=\frac{1+t^{11}}{1+t} \quad c_{23}(t)=\frac{1-t^{23}}{1-t} \quad c_{24}(t)=\frac{1+t^{12}}{1+t^{4}} \\
& c_{25}(t)=\frac{1-t^{25}}{1-t^{5}} \quad c_{26}(t)=\frac{1+t^{13}}{1+t} \quad c_{27}(t)=\frac{1-t^{27}}{1-t^{9}} \\
& c_{28}(t)=\frac{1+t^{14}}{1+t^{2}} \quad c_{29}(t)=\frac{1-t^{29}}{1-t} \quad c_{30}(t)=\frac{\left(1+t^{15}\right)(1+t)}{\left(1+t^{3}\right)\left(1+t^{5}\right)}
\end{aligned}
$$


Lemma 1 (Gauss). Irreducible polynomials whose roots are roots of unity are precisely the collection of cyclotomic polynomials.

Here are some elementary properties of the cyclotomic polynomials (cf. [18]).

(p1) If $p>1$ is prime then $c_{p}(t)=\left(1-t^{p}\right) /(1-t)$.

(p2) If $p=2 r$ with $r$ odd then $c_{2 r}(t)=c_{r}(-t)$.

(p3) If $p=2^{\alpha}$ with $\alpha$ a positive integer, then $c_{p}(t)=1+t^{2^{\alpha-1}}$.

(p4) If $p=r^{\alpha}$ with $r>2$ prime and $\alpha$ a positive integer,, then $c_{p}(t)=c_{r}\left(t^{r^{\alpha-1}}\right)=$ $\left(1-t^{r^{\alpha}}\right) /\left(1-t^{r^{\alpha-1}}\right)$.

(p5) If $p=2^{n} r$ with $r$ odd and $n>1$, then $c_{n}(t)=c_{2 r}\left(t^{2^{n-1}}\right)$.

(p6) For all $n$ we have that $c_{n}(0)=1$, and the leading term of $c_{n}(t)$ is 1 if $n \geq 2$.

(p7) The degree of $c_{n}(t)$ is even for $n>2$.

\section{Lefschetz Zeta Function}

If $f: M \rightarrow M$ is a continuous map on a compact $n$-dimensional topological manifold (orientable or not), it induces a homomorphism on the $k$-th rational homology group of $M$ for $0 \leq k \leq n$, i.e. $f_{* k}: H_{k}(M, \mathbb{Q}) \rightarrow H_{k}(M, \mathbb{Q})$. The $H_{k}(M, \mathbb{Q})$ is a finite dimensional vector space over $\mathbb{Q}$ and $f_{* k}$ is a linear map whose matrix has integer entries, then Lefschetz number of $f$ is defined as

$$
L(f)=\sum_{k=0}^{n}(-1)^{k} \operatorname{trace}\left(f_{* k}\right) .
$$

The Lefschetz Fixed Point Theorem states that if $L(f) \neq 0$ then $f$ has a fixed point (cf. [6]).

The Lefschetz zeta function of $f$ is defined as

$$
\zeta_{f}(t)=\exp \left(\sum_{m \geq 1} \frac{L\left(f^{m}\right)}{m} t^{m}\right) .
$$

This function keeps the information of the Lefschetz number for all the iterates of $f$, so this function gives information about the set of periods of $f$. There is an alternative way to compute it:

$$
\zeta_{f}(t)=\prod_{k=0}^{n} \operatorname{det}\left(I d_{* k}-t f_{* k}\right)^{(-1)^{k+1}},
$$

where $n=\operatorname{dim} M, n_{k}=\operatorname{dim} H_{k}(M, \mathbb{Q}), I d:=I d_{* k}$ is the identity map on $H_{k}(M, \mathbb{Q})$, and by convention $\operatorname{det}\left(I d_{* k}-t f_{* k}\right)=1$ if $n_{k}=0$ (cf. [10]).

In [21] we computed the function $\zeta_{f}(t)$ in the case of Morse-Smale diffeomorphisms on orientable compact surfaces without boundary. Here we are interested in computing the function $\zeta_{f}(t)$ when $f$ is a Morse-Smale diffeomorphism on a non-orientable compact surface without boundary of genus $g$, i.e. $M=N_{g}$ for any $g \geq 1$. We recall that $H_{k}(M, \mathbb{Q})$ is a vector space over $\mathbb{Q}$, so it is always torsion free. For non-orientable surfaces $H_{0}(M, \mathbb{Q})=\mathbb{Q}$ and $H_{2}(M, \mathbb{Q}) \approx 0$. If $M=N_{g}$ for $g \geq 1$, i.e. a connected sum of $g$ real projective planes, then

$$
H_{1}(M, \mathbb{Q})=\underbrace{\mathbb{Q} \oplus \cdots \oplus \mathbb{Q}}_{g-1} .
$$


A rational linear transformation is called quasi-unipotent if their eigenvalues are root of unity. We say that a continuous map $f: M \rightarrow M$ is quasi-unipotent if the maps $f_{* k}$ are quasi-unipotent, or $0 \leq k \leq n$, where $n$ is the dimension of the manifold $M$.

Proposition 2 (Shub [28]). Let $M$ be a compact manifold. If $f: M \rightarrow M$ is a Morse-Smale diffeomorphism, then $f$ is quasi-unipotent.

Suppose the rational linear transformation $f_{* 1}$ is quasi-unipotent. Then the characteristic polynomial is in $\mathbb{Z}[x]$ and its factors over $\mathbb{Z}$ are irreducible polynomials whose roots are roots of unity. So these factors are cyclotomic polynomials.

The following result is well known for a proof see [21].

Proposition 3. If $f_{*_{1}}$ is quasi-unipotent, then

$$
\operatorname{det}\left(I d_{* 1}-t f_{* 1}\right)=(-1)^{1+\operatorname{det}\left(f_{* 1}\right)} \operatorname{det}\left(f_{* 1}-t I d_{* 1}\right) .
$$

Proposition 4. Let $M$ be a non-orientable compact surface without boundary and $f: M \rightarrow M$ be a differentiable map. Suppose that $f_{* 1}$ is quasi-unipotent with characteristic polynomial $p(t)$. Then

$$
\zeta_{f}(t)=\frac{p(t)}{1-t}
$$

Proof. From Proposition 3 follows that for computing all the possible values of $\operatorname{det}\left(I d_{* 1}-\right.$ $\left.t f_{* 1}\right)$ for a certain $n_{1}$ fixed, it is sufficient to consider the product of all cyclotomic polynomials such that the degree of the product is equal to $n_{1}$. Since $f_{* 0}=1$ and $H_{2}(M, \mathbb{Q}) \approx 0$, we can compute the Lefschetz zeta functions for $f$, using formula (2).

We say that a rational function $\zeta(t)$ is a realizable or allowed zeta function, if $\zeta(t)$ satisfies formula (3) for a map $f: M \rightarrow M$ satisfying the hypothesis of Proposition 4.

Since $H_{1}\left(N_{g}, \mathbb{Q}\right)$ is $g-1$ dimensional vector space; the characteristic polynomial of $f_{* 1}$, for a quasi-unipotent map $f$, is a product of cyclotomic polynomials of total degree $g-1$. So Proposition 4 can be stated as follows.

Proposition 5. Let $g \geq 1$ and $p(t)$ be a product of cyclotomic polynomials of total degree $g-1$. Then the rational function $p(t)(1-t)^{-1}$ is a Lefschetz zeta function $\zeta_{f}(t)$ for some $C^{1}$ quasi-unipotent map $f: N_{g} \rightarrow N_{g}$.

If $g=1$ then $N_{1}$ is the real projective plane, then the only possible zeta function is $\zeta_{f}(t)=(1-t)^{-1}$. For $N_{2}$, the Klein Bottle the possible Lefschetz zeta functions are $\zeta_{f}(t)=1$ or $\zeta_{f}(t)=(1+t)(1-t)^{-1}$. For $N_{3}$ the allowed Lefschetz zeta functions are

$$
1-t, \quad 1+t, \quad \frac{(1+t)^{2}}{1-t}, \quad \frac{1-t^{3}}{(1-t)^{2}}, \quad \frac{1+t^{3}}{(1-t)(1+t)}, \quad \frac{1+t^{2}}{1-t} .
$$

In Tables 2, 3, 4, 5 and 6 are listed the allowed Lefschetz zeta functions on $N_{g}$ for $g=4,5,6,7$.

\section{The minimal Set of Lefschetz Periods $\operatorname{MPER}_{L}(f)$}

Let $M$ be a $C^{1}$ compact manifold and let $f: M \rightarrow M$ be a $C^{1}$ map. Let $x$ be a hyperbolic periodic point of period $p$ of $f$ and $E_{x}^{u}$ its unstable space, i.e. the subspace of the tangent space $T_{x} M$ generated by the eigenvectors of $D f^{p}(x)$ of modulus larger than 1. Let $\gamma$ be the orbit of $x$, the index $u$ of $\gamma$ is the dimension of $E_{x}^{u}$. We define 
TABLE 2. The allowed Lefschetz zeta functions on $N_{4}$.

$$
\begin{aligned}
& (1-t)^{2}, \quad(1-t)(1+t), \quad(1+t)^{2}, \quad \frac{(1+t)^{3}}{1-t}, \quad \frac{1-t^{3}}{1-t}, \\
& \frac{1+t^{3}}{1+t}, \quad 1+t^{2}, \quad \frac{(1+t)\left(1-t^{3}\right)}{(1-t)^{2}}, \quad \frac{(1+t)\left(1+t^{2}\right)}{1-t} \cdot \frac{1+t^{3}}{1-t}
\end{aligned}
$$

TABLE 3. The allowed Lefschez zeta functions on $N_{5}$.

\begin{tabular}{cccc}
\hline$\frac{1-t^{5}}{(1-t)^{2}}$, & $\frac{1+t^{5}}{(1-t)(1+t)}$, & $\frac{1+t^{4}}{1-t}$, & $\frac{\left(1-t^{3}\right)^{2}}{(1-t)^{3}}$ \\
$\frac{\left(1-t^{3}\right)\left(1+t^{3}\right)}{(1+t)(1-t)^{2}}$, & $\frac{\left(1-t^{3}\right)\left(1+t^{2}\right)}{(1-t)^{2}}$, & $\frac{\left(1+t^{3}\right)^{2}}{(1+t)^{2}(1-t)}$, & $\frac{\left(1+t^{3}\right)\left(1+t^{2}\right)}{(1+t)(1-t)}$ \\
$\frac{\left(1+t^{2}\right)^{2}}{1-t}$, & $\frac{1+t^{6}}{(1-t)\left(1+t^{2}\right)}$ & $\frac{\left(1-t^{3}\right)(1+t)}{1-t}$, & $\frac{\left(1-t^{3}\right)(1+t)^{2}}{(1-t)^{2}}$ \\
$\frac{\left(1+t^{3}\right)(1-t)}{1+t}$, & $1+t^{3}$, & $\frac{\left(1+t^{3}\right)(1+t)}{1-t}$, & $1-t^{3}$, \\
$\left(1+t^{2}\right)(1-t)$, & $\left(1+t^{2}\right)(1+t)$, & $\frac{\left(1+t^{2}\right)(1+t)^{2}}{1-t}$, & $(1+t)^{3}$ \\
$(1+t)(1-t)^{2}$, & $(1+t)^{2}(1-t)$, & $(1+t)^{3}$, & $1-t$
\end{tabular}

the orientation type $\Delta$ of $\gamma$ as +1 if $D f^{p}(x): E_{x}^{u} \rightarrow E_{x}^{u}$ preserves orientation and -1 if reverses the orientation. The collection of the triples $(p, u, \Delta)$ belonging to all periodic orbits of $f$ is called the periodic data of $f$. The same triple can appear more than once if it corresponds to different periodic orbits.

Theorem 6 (Franks [9]). Let $f$ be a $C^{1}$ a map on a compact manifold without boundary having finitely many periodic points all of them hyperbolic, and let $\Sigma$ be the periodic data of $f$. Then the Lefschetz zeta function $\zeta_{f}(t)$ of $f$ satisfies

$$
\zeta_{f}(t)=\prod_{(p, u, \Delta) \in \Sigma}\left(1-\Delta t^{p}\right)^{(-1)^{u+1}} .
$$

Clearly the Morse-Smale diffeomorphisms on non-orientable compact surfaces without boundary satisfy the hypotheses of this theorem.

Theorem 6 allow to define the minimal set of Lefschetz periods for a $C^{1}$ map on a compact manifold having finitely many periodic points all of them hyperbolic. Such a map has a Lefschetz zeta function of the form (5). Note that in general the expression of one of these Lefschetz zeta functions is not unique as product of the elements of 
TABLE 4. The allowed Lefschez zeta functions on $N_{6}$.

\begin{tabular}{cccc}
$\frac{1-t^{5}}{1-t}$, & $\frac{1+t^{5}}{1+t}$, & $1+t^{4}$, & $\frac{\left(1-t^{3}\right)^{2}}{(1-t)^{2}}$, \\
$\frac{\left(1-t^{5}\right)(1+t)}{(1-t)^{2}}$, & $\frac{1+t^{5}}{1-t}$, & $\frac{\left(1+t^{4}\right)(1+t)}{1-t}$, & $\frac{\left(1+t^{6}\right)(1+t)}{\left(1+t^{2}\right)(1-t)}$ \\
$\frac{1+t^{6}}{1+t^{2}}$, & $\frac{\left(1-t^{3}\right)\left(1+t^{2}\right)}{1-t}$, & $\frac{\left(1+t^{3}\right)^{2}}{(1+t)^{2}}$, & $\frac{\left(1+t^{3}\right)\left(1+t^{2}\right)}{1+t}$, \\
$\frac{\left(1-t^{3}\right)\left(1+t^{3}\right)}{(1-t)^{2}}$, & $\frac{\left(1-t^{3}\right)\left(1+t^{2}\right)(1+t)}{(1-t)^{2}}$, & $\frac{\left(1+t^{3}\right)^{2}}{(1+t)(1-t)}$, & $\frac{\left(1+t^{2}\right)^{2}(1+t)}{1-t}$, \\
$\frac{\left(1-t^{3}\right)^{2}(1+t)}{(1-t)^{3}}$, & $\frac{\left(1-t^{3}\right)\left(1+t^{3}\right)}{(1+t)(1-t)}$, & $\left(1-t^{3}\right)(1+t)$, & $\left(1-t^{3}\right)(1+t)$, \\
$\frac{\left(1+t^{3}\right)(1-t)^{2}}{1+t}$, & $\left(1+t^{3}\right)(1-t)$, & $\frac{\left(1-t^{3}\right)(1+t)^{2}}{1-t}$, & $\frac{\left(1-t^{3}\right)(1+t)^{3}}{(1-t)^{2}}$, \\
$\frac{\left(1+t^{3}\right)(1+t)^{2}}{1-t}$, & $\left(1-t^{3}\right)(1-t)$, & $\left(1+t^{3}\right)(1+t)$, & $\left(1+t^{2}\right)(1-t)^{2}$, \\
$\left(1+t^{2}\right)^{2}$, & $\frac{\left(1+t^{2}\right)(1+t)^{3}}{1-t}$, & $\frac{(1+t)^{5}}{1-t}$, & $\left(1+t^{2}\right)(1+t)(1-t)$, \\
$(1+t)^{2}(1-t)^{2}$, & $(1-t)^{3}(1+t)$, & $\left(1+t^{2}\right)(1+t)^{2}$, & $(1-t)^{4}$, \\
$(1+t)^{3}(1-t)$, & $(1+t)^{4}$. & & \\
\hline
\end{tabular}

the form $\left(1 \pm t^{p}\right)^{ \pm 1}$. For instance the following possible Lefschetz zeta function can be written in four different ways in the form given by (5):

$$
\zeta_{f}(t)=\frac{\left(1-t^{3}\right)\left(1+t^{3}\right)}{(1-t)^{2}(1+t)}=\frac{1-t^{6}}{(1-t)^{2}(1+t)}=\frac{1-t^{6}}{(1-t)\left(1-t^{2}\right)}=\frac{\left(1-t^{3}\right)\left(1+t^{3}\right)}{(1-t)\left(1-t^{2}\right)} .
$$

According with Theorem 6 , the first expression will provide the periods $\{1,3\}$ for $f$, the second the periods $\{1,6\}$, the third the period $\{1,2,6\}$, and finally the fourth the periods $\{1,2,3\}$. Then for this Lefschetz zeta function $\zeta_{f}(t)$ we will define its minimal set of Lefschetz periods as

$$
\operatorname{MPer}_{L}(f)=\{1,3\} \cap\{1,6\} \cap\{1,2,6\} \cap\{1,2,3\}=\{1\} .
$$

If $\zeta_{f}(t) \neq 1$ then it can be written as

$$
\zeta_{f}(t)=\prod_{i=1}^{N_{\zeta}}\left(1+\Delta_{i} t^{r_{i}}\right)^{m_{i}},
$$


TABLE 5. The allowed Lefschez zeta functions on $N_{7}$.

$$
\begin{aligned}
& \frac{1-t^{7}}{(1-t)^{2}} \\
& \frac{1-t^{9}}{\left(1-t^{3}\right)(1-t)} \\
& \frac{1+t^{7}}{(1+t)(1-t)} \\
& \frac{1+t^{9}}{\left(1+t^{3}\right)(1-t)}, \\
& \frac{\left(1-t^{5}\right)\left(1-t^{3}\right)}{(1-t)^{3}}, \quad \frac{\left(1-t^{5}\right)\left(1+t^{3}\right)}{(1-t)^{2}(1+t)}, \\
& \frac{\left(1-t^{5}\right)\left(1+t^{2}\right)}{(1-t)^{2}} \\
& \frac{\left(1+t^{5}\right)\left(1-t^{3}\right)}{(1+t)(1-t)^{2}}, \\
& \frac{\left(1+t^{5}\right)\left(1+t^{3}\right)}{(1+t)^{2}(1-t)}, \quad \frac{\left(1+t^{5}\right)\left(1+t^{2}\right)}{(1-t)(1+t)}, \\
& \frac{\left(1+t^{4}\right)\left(1-t^{3}\right)}{(1-t)^{2}} \\
& \frac{\left(1+t^{4}\right)\left(1+t^{3}\right)}{(1-t)(1+t)} \\
& \frac{\left(1+t^{4}\right)\left(1+t^{2}\right)}{1-t}, \quad \frac{\left(1+t^{6}\right)(1-t)}{1+t^{2}} \\
& \frac{\left(1+t^{6}\right)(1+t)}{1+t^{2}} \\
& \frac{\left(1+t^{6}\right)(1+t)^{2}}{\left(1+t^{2}\right)(1-t)} \\
& \frac{\left(1+t^{6}\right)\left(1-t^{3}\right)}{\left(1+t^{2}\right)(1-t)^{2}}, \quad \frac{1+t^{6}}{1-t}, \\
& \frac{\left(1+t^{6}\right)\left(1+t^{3}\right)}{\left(1+t^{2}\right)(1-t)(1+t)}, \\
& \frac{\left(1-t^{5}\right)(1+t)^{2}}{(1-t)^{2}} \\
& \left(1+t^{4}\right)(1-t), \quad\left(1+t^{4}\right)(1+t), \\
& 1-t^{5}, \\
& 1+t^{5}, \\
& \frac{\left(1+t^{5}\right)(1+t)}{1-t} \\
& \frac{\left(1-t^{5}\right)(1+t)}{1-t}, \\
& \frac{\left(1+t^{5}\right)(1-t)}{1+t}, \\
& \frac{\left(1+t^{4}\right)(1+t)^{2}}{1-t} \\
& \frac{\left(1-t^{3}\right)^{2}(1+t)^{2}}{(1-t)^{3}}, \quad \frac{\left(1-t^{3}\right)\left(1+t^{3}\right)}{1+t}, \\
& \frac{\left(1-t^{3}\right)^{3}}{(1-t)^{4}} \\
& \frac{\left(1-t^{3}\right)^{2}\left(1+t^{3}\right)}{(1-t)^{3}(1+t)}, \\
& \frac{\left(1-t^{3}\right)^{2}\left(1+t^{2}\right)}{(1-t)^{3}}, \quad \frac{\left(1-t^{3}\right)\left(1+t^{3}\right)^{2}}{(1-t)^{2}(1+t)^{2}} \\
& \frac{\left(1-t^{3}\right)\left(1+t^{3}\right)\left(1+t^{2}\right)}{(1-t)^{2}(1+t)} \\
& \frac{\left(1+t^{3}\right)\left(1+t^{2}\right)^{2}}{(1+t)(1-t)} \\
& \frac{\left(1-t^{3}\right)\left(1+t^{2}\right)^{2}}{(1-t)^{2}} \\
& \frac{\left(1+t^{3}\right)^{3}}{(1-t)(1+t)^{3}} \\
& \frac{\left(1+t^{2}\right)\left(1+t^{3}\right)^{2}}{(1-t)(1+t)^{2}} \\
& \frac{\left(1+t^{2}\right)^{3}}{1-t} \\
& \frac{\left(1-t^{3}\right)^{2}}{1-t} \\
& \frac{\left(1-t^{3}\right)^{2}(1+t)}{(1-t)^{2}} \\
& \left(1+t^{2}\right)^{2}(1-t) \\
& \left(1+t^{2}\right)(1+t),
\end{aligned}
$$

where $\Delta_{i}= \pm 1$, the $r_{i}$ 's are positive integers, $m_{i}$ 's are nonzero integers and $N_{\zeta}$ is a positive integer depending on $f$.

If $\zeta_{f}(t) \neq 1$ the minimal set of Lefschetz periods of $f$ is defined as

$$
\operatorname{MPer}_{L}(f):=\bigcap\left\{r_{1}, \ldots, r_{N_{\zeta}}\right\}
$$

where the intersection is considered over all the possible expressions $(6)$ of $\zeta_{f}(t)$. If $\zeta_{f}(t)=1$ then we define $\operatorname{MPer}_{L}(f):=\emptyset$. Roughly speaking the minimal set of Lefschetz periods of $f$ is the intersection of all the sets of periods forced by the finitely many different representations of $\zeta_{f}(t)$ as products of the form $\left(1 \pm t^{p}\right)^{ \pm 1}$. Clearly

$$
\operatorname{MPer}_{L}(f) \subseteq \operatorname{Per}(f)
$$


TABLE 6. The allowed Lefschez zeta functions on $N_{7}$ (cont.).

$$
\begin{array}{llll}
\frac{\left(1-t^{3}\right)\left(1+t^{3}\right)(1+t)}{(1-t)^{2}}, & \frac{\left(1-t^{3}\right)\left(1+t^{3}\right)}{1-t}, & \left(1-t^{3}\right)\left(1+t^{2}\right), & \frac{\left(1-t^{3}\right)(1+t)^{3}}{1-t}, \\
\frac{\left(1-t^{3}\right)\left(1+t^{2}\right)(1+t)^{2}}{(1-t)^{2}}, & \frac{\left(1+t^{3}\right)^{2}(1-t)}{(1+t)^{2}}, & \frac{\left(1+t^{2}\right)^{2}(1+t)^{2}}{1-t}, & \frac{\left(1+t^{3}\right)^{2}}{1-t}, \\
\frac{\left(1+t^{3}\right)\left(1+t^{2}\right)(1-t)}{1+t}, & \left(1-t^{3}\right)(1-t)^{2}, & \left(1+t^{3}\right)\left(1+t^{2}\right), & \frac{\left(1+t^{3}\right)^{2}}{1+t}, \\
\frac{\left(1+t^{3}\right)\left(1+t^{2}\right)(1+t)}{1-t}, & \frac{\left(1-t^{3}\right)(1+t)^{4}}{(1-t)^{2}}, & \left(1-t^{3}\right)(1+t)^{2}, & \left(1+t^{3}\right)(1+t)^{2}, \\
\left(1-t^{3}\right)(1-t)(1+t), & \frac{\left(1+t^{3}\right)(1-t)^{3}}{1+t}, & \left(1+t^{3}\right)(1-t)^{2}, & (1+t)^{5}, \\
\left(1+t^{2}\right)(1+t)(1-t)^{2}, & \frac{\left(1+t^{3}\right)(1+t)^{3}}{1-t}, & \left(1+t^{2}\right)(1-t)^{3}, & (1-t)^{5}, \\
\left(1+t^{2}\right)(1-t)(1+t)^{2}, & \left(1+t^{2}\right)(1+t)^{3}, & \frac{\left(1+t^{2}\right)(1+t)^{4}}{1-t}, & \frac{(1+t)^{6}}{1-t}, \\
\left(1+t^{3}\right)(1-t)(1+t), & (1-t)^{4}(1+t), & (1-t)^{3}(1+t)^{2}, & (1-t)^{2}(1+t)^{3}, \\
\left(1-t^{3}\right)\left(1+t^{2}\right)(1+t) & (1-t)(1+t)^{4} . &
\end{array}
$$

The results of this article apply to the class of maps that satisfies Theorem 6 and Proposition 4, i.e. $C^{1}$ maps on non-orientable compact surfaces without boundary, such that there action on the first homology group $f_{* 1}$ is quasi-unipotent and have finitely many periodic points all of them hyperbolic. By Proposition 2 the MorseSmale diffeomorphisms are in this class.

The following result was proved in [22], since it is important in the present work and its proof is easy we provide it for completeness.

Proposition 7. There are no even numbers in $\operatorname{MPer}_{L}(f)$.

Proof. If the number $2 d$ is in $\operatorname{MPer}_{L}(f)$ then $\left(1 \pm t^{2 d}\right)^{m}$ is a factor of the Lefschetz zeta function $\zeta_{f}(t)$, for some $m \neq 0$. So if the factor is $\left(1-t^{2 d}\right)^{m}$ it can be written as $\left(1-t^{d}\right)^{m}\left(1+t^{d}\right)^{m}$, since the intersection of the exponents is taken over all possible expressions (6) of $\zeta_{f}(t)$, the number $2 d$ is not in $\operatorname{MPer}_{L}(f)$.

If the factor is $\left(1+t^{2 d}\right)^{m}$, then it can be written as

$$
\left(1+t^{2 d}\right)^{m}=\frac{\left(1+t^{2 d}\right)^{m}\left(1-t^{2 d}\right)^{m}}{\left(1-t^{2 d}\right)^{m}}=\frac{\left(1-t^{4 d}\right)^{m}}{\left(1-t^{d}\right)^{m}\left(1+t^{d}\right)^{m}} .
$$

Therefore $2 d \notin \operatorname{MPer}_{L}(f)$. 


\section{5. $\operatorname{MPer}_{L}(f)$ For the Morse-Smale Diffeomorphisms on $N_{g}$}

One of our main results is the following.

Theorem 8. Let $f$ be a Morse-Smale diffeomorphism on $N_{g}$.

(a) If $g=1$ then $\operatorname{MPer}_{L}(f)$ is $\{1\}$.

(b) If $g=2$ then $\operatorname{MPer}_{L}(f)$ is $\emptyset$ or $\{1\}$.

(c) If $g=3$ then $M \operatorname{Per}_{L}(f)$ is $\{1\},\{3\}$ or $\{1,3\}$.

(d) If $g=4$ then $M \operatorname{Per}_{L}(f)$ is $\emptyset,\{1\}$ or $\{1,3\}$.

(e) If $g=5$ then $M \operatorname{Per}_{L}(f)$ is $\{1\},\{3\},\{5\},\{1,3\}$ or $\{1,5\}$.

(f) If $g=6$ then $\operatorname{MPer}_{L}(f)$ is $\emptyset,\{1\},\{3\},\{1,3\}$ or $\{1,5\}$.

(g) If $g=7$ then $\operatorname{MPer}_{L}(f)$ is $\{1\},\{3\},\{5\},\{7\},\{1,3\},\{1,5\}\{1,7\},\{1,3,5\}$ or $\{1,3,9\}$.

(h) If $g=8$ then $\operatorname{MPer}_{L}(f)$ is $\emptyset,\{1\},\{3\},\{1,3\},\{1,5\},\{1,7\},\{3,5\},\{3,9\}$, $\{1,3,5\}$ or $\{1,3,9\}$.

(i) If $g=9$ then $\operatorname{MPer}_{L}(f)$ is $\{1\},\{3\},\{5\},\{7\},\{9\},\{1,3\},\{1,5\}\{1,7\},\{1,9\}$, $\{3,9\},\{1,3,5\},\{1,3,7\},\{1,3,9\},\{3,5,15\}$ or $\{1,3,5,15\}$.

Proof. If $g=1$, from section 3 we know that $\zeta_{f}(t)=(1-t)^{-1}$ is the only allowed Lefschetz zeta function for the real projective plane. $\operatorname{So~}^{M_{P e r}}(f)=\{1\}$.

If $g=2$ then the allowed Lefschetz zeta functions for $N_{2}$ (the Klein bottle), again from section 3 are either $\zeta_{f}(t)=1$, or $\zeta_{f}(t)=(1+t)(1-t)^{-1}$. In the former case its $\operatorname{MPer}_{L}(f)$ is the empty set, and in the later on is $\operatorname{MPer}_{L}(f)=\{1\}$.

For the genus $g=3$ the 6 allowed Lefschetz zeta functions $\zeta_{f}(t)$ are described in (4). From these zeta functions the unique $\operatorname{MPer}_{L}(f)$ are $\{1\},\{3\}$ and $\{1,3\}$ coming, for instance, from the Lefschetz zeta functions $1-t,\left(1+t^{3}\right) /((1-t)(1+t))$ and $\left(1-t^{3}\right) /(1-t)^{2}$, respectively.

In Table 2 are listed the 10 allowed Lefschetz zeta functions for $N_{4}$. From these zeta functions the unique $\operatorname{MPer}_{L}(f)$ are $\emptyset,\{1\}$ and $\{1,3\}$ coming, for instance, from the Lefschetz zeta functions $1+t^{2},(1-t)^{2}$ and $\left(1-t^{3}\right) /(1-t)$, respectively.

The 24 allowed Lefschetz zeta functions for $N_{5}$ are listed in Table 3 . We get that $\operatorname{MPer}_{L}(f)$ must be $\{1\},\{3\},\{5\},\{1,3\}$ or $\{1,5\}$ coming, for instance, from $(1+t)^{3}$, $1-t^{3},\left(1+t^{5}\right) /((1-t)(1+t)),\left(1+t^{3}\right)(1-t) /(1+t)$ and $\left(1-t^{5}\right) /(1-t)^{2}$, respectively.

For the genus $g=6$ the 38 allowed Lefschetz zeta functions $\zeta_{f}(t)$ are described in Table 4. From these zeta functions the unique $\operatorname{MPer}_{L}(f)$ are $\emptyset,\{1\},\{3\},\{1,3\}$ and $\{1,5\}$ coming, for instance, from the Lefschetz zeta functions $1+t^{4},(1-t)^{4}$, $\left(1+t^{3}\right)^{2} /((1-t)(1+t)),\left(1-t^{3}\right)(1-t)$ and $\left(1-t^{5}\right) /(1-t)$, respectively.

The 78 allowed Lefschetz zeta functions for $N_{7}$ are listed in Table 5 . We get that $\operatorname{MPer}_{L}(f)$ must be $\{1\},\{3\},\{5\},\{7\},\{1,3\},\{1,5\}\{1,7\},\{1,3,5\}$ and $\{1,3,9\}$ coming, for instance, from $(1+t)^{5},\left(1+t^{4}\right)\left(1+t^{3}\right) /((1-t)(1+t)),\left(1+t^{5}\right)\left(1+t^{2}\right) /((1-$ $t)(1+t)),\left(1+t^{7}\right) /((1-t)(1+t)),\left(1+t^{4}\right)\left(1-t^{3}\right) /(1-t)^{2},\left(1-t^{5}\right)\left(1+t^{2}\right) /(1-t)^{2}$, $\left(1-t^{7}\right) /(1-t)^{2},\left(1-t^{5}\right)\left(1-t^{3}\right) /(1-t)^{3}$ and $\left(1-t^{9}\right) /\left(\left(1-t^{3}\right)(1-t)\right)$, respectively.

The cases $g=8$ and $g=9$ can be proved in a similar way, i.e. first computing all the Lefschetz zeta function allowed for $N_{8}$ and $N_{9}$, and after checking that the unique sets $\operatorname{MPer}_{L}(f)$ that they provide are the ones described in statements (h) and (i). In fact, for genus $g=8$ the sets $\emptyset,\{1\},\{3\},\{1,3\},\{1,5\},\{1,7\},\{3,5\},\{3,9\},\{1,3,5\}$ can $\{1,3,9\}$ come, for instance, from $(1-t)^{3}(1+t)^{3},(1+t)^{6},\left(1+t^{3}\right)^{2}\left(1+t^{2}\right) /((1-t)(1+t))$, $\left(1+t^{9}\right)\left(1-t^{3}\right) /(1-t),\left(1-t^{5}\right)\left(1+t^{2}\right) /(1-t),\left(1-t^{7}\right) /(1-t),\left(1+t^{5}\right)\left(1+t^{3}\right) /((1-t)(1+t))$, $\left(1+t^{9}\right) /\left(1+t^{3}\right),\left(1-t^{3}\right)\left(1-t^{5}\right) /(1-t)^{2}$ and $\left(1-t^{9}\right)(1+t) /\left(\left(1-t^{3}\right)(1-t)\right)$; and 
for genus $g=9$ the sets $\{1\},\{3\},\{5\},\{7\},\{9\},\{1,3\},\{1,5\}\{1,7\},\{1,9\},\{3,9\}$, $\{1,3,5\},\{1,3,7\},\{1,3,9\},\{3,5,15\}$ and $\{1,3,5,15\}$ come, for instance, from $(1+t)^{7}$, $\left(1+t^{4}\right)\left(1+t^{3}\right)\left(1+t^{2}\right) /((1-t)(1+t)),\left(1+t^{5}\right)\left(1+t^{2}\right)^{2} /((1-t)(1+t)),\left(1+t^{7}\right)\left(1+t^{2}\right) /((1-$ $t)(1+t)),\left(1+t^{9}\right) /((1-t)(1+t)),\left(1+t^{4}\right)\left(1-t^{3}\right)\left(1+t^{2}\right) /(1-t)^{2},\left(1-t^{5}\right)\left(1+t^{2}\right)^{2} /(1-t)^{2}$, $\left(1-t^{7}\right)\left(1+t^{2}\right) /(1-t)^{2},\left(1-t^{9}\right) /(1-t)^{2},\left(1-t^{9}\right)\left(1+t^{3}\right) /\left(\left(1-t^{3}\right)(1+t)(1-t)\right)$, $\left(1-t^{5}\right)\left(1-t^{3}\right) /(1-t),\left(1-t^{7}\right)\left(1-t^{3}\right) /(1-t)^{3}$, and $\left(1-t^{9}\right)\left(1+t^{2}\right) /\left(\left(1-t^{3}\right)(1-t)\right)$, $\left(1-t^{15}\right) /\left(\left(1-t^{3}\right)\left(1-t^{5}\right)\right)$ and $\left(1+t^{15}\right)(1+t) /\left(\left(1+t^{3}\right)\left(1+t^{5}\right)(1-t)\right)$, respectively.

We denote by $\operatorname{Diff}\left(N_{g}\right)$ the set of all $C^{1}$ Morse-Smale diffeomorphisms on the non-orientable compact surface without boundary $N_{g}$ of genus $g$.

In what follows we study what kind of subsets of positive integers can be minimal sets of Lefschetz periods for the $C^{1}$ Morse-Smale diffeomorphisms on a non-orientable compact surface without boundary.

Theorem 9. The following statement hold.

(a) If $g$ is an odd prime, then there exists $f_{1}, f_{2} \in \operatorname{Diff}\left(N_{g}\right)$ such that $\operatorname{MPer}_{L}\left(f_{1}\right)=$ $\{1, g\}$, and $\operatorname{MPer}_{L}\left(f_{2}\right)=\{g\}$.

(b) If $g=p^{\alpha-1}(p-1)+1$ with $p$ an odd prime and $\alpha>1$, then there exists $f \in \operatorname{Diff}\left(N_{g}\right)$ such that $\operatorname{MPer}_{L}(f)=\left\{1, p^{\alpha-1}, p^{\alpha}\right\}$.

(c) If $g$ is even, then there exists $f \in \operatorname{Diff}\left(N_{g}\right)$ such that $\operatorname{MPer}_{L}(f)=\emptyset$.

(d) If $g$ is odd, then $\operatorname{MPer}_{L}(f) \neq \emptyset$ for all $f \in \operatorname{Diff}\left(N_{g}\right)$.

(e) For all positive integer $g$ there exists $f \in \operatorname{Diff}\left(N_{g}\right)$ such that $\operatorname{MPer}_{L}(f)=\{1\}$.

(f) If $g$ is odd and $p$ is an odd prime such that $1<p \leq g$, then there exists $f \in \operatorname{Diff}\left(N_{g}\right)$ such that $\operatorname{MPer}_{L}(f)=\{p\}$.

(g) Let $p_{1}, \ldots, p_{k}$ be different odd primes larger than 1 , and let $\alpha_{1}, \ldots, \alpha_{k}$ positive integers.

(g.1) Then there exists $f \in \operatorname{Diff}\left(N_{g}\right)$ such that $\operatorname{MPer}_{L}(f)=\left\{p_{1}, \ldots, p_{k}\right\}$, with $g=\left(\sum_{i+1}^{k} p_{i}\right)-(k-1)$ if $k$ is odd, and $g=\left(\sum_{i+1}^{k} p_{i}\right)-(k-2)$ if $k$ is

(g.2) Then there exists $f \in \operatorname{Diff}\left(N_{g}\right)$ with a convenient genus $g$ such that $\operatorname{MPer}_{L}(f)=\left\{p_{1}^{\alpha_{1}}, \ldots, p_{k}^{\alpha_{k}}\right\}$.

(g.3) Let $p$ be and odd prime number. Then there exists $f \in \operatorname{Diff}\left(N_{g}\right)$ with a convenient genus $g$ such that $\operatorname{MPer}_{L}(f)=\left\{p^{\alpha_{1}}, \ldots, p^{\alpha_{k}}\right\}$.

(g.4) Let $\alpha_{i, j}$ positive integers such that $\alpha_{i, j}>\alpha_{i, j+1}$ for $1 \leq i \leq k$ and $1 \leq j \leq l_{i}$. Then there exists $f \in \operatorname{Diff}\left(N_{g}\right)$ with a convenient genus $g$ such that $\operatorname{MPer}_{L}(f)=\left\{p_{1}^{\alpha_{1,1}}, \ldots, p_{1}^{\alpha_{1, l_{1}}}, \ldots, p_{k}^{\alpha_{k, 1}}, \ldots, p_{k}^{\alpha_{k, l_{k}}}\right\}$.

Statement (d) of Theorem 9 shows an important difference in the periodic structure between the $C^{1}$ Morse-Smale diffeomorphisms on orientable and non-orientable compact surfaces without boundary. In [17] it was shown that for all orientable compact surfaces without boundary there are $C^{1}$ Morse-Smale diffeomorphisms having their minimal set of Lefschetz periods empty, and statement (d) shows that this is not the case for $C^{1}$ Morse-Smale diffeomorphisms on the non-orientable compact surfaces without boundary.

The results of statements (f) and (g) of Theorem 9 also hold for $C^{1}$ Morse-Smale diffeomorphisms on orientable compact surfaces without boundary, and their proof are similar, see [17]. 
Proof of Theorem 9(a). Let $p(t)=c_{g}(t)$, by property (p1) of the cyclotomic polynomials, its degree is $g-1$. So

$$
\zeta_{f}(t)=\frac{p(t)}{1-t}=\frac{c_{g}(t)}{c_{1}(t)}=\frac{1-t^{g}}{(1-t)^{2}},
$$

and by Proposition $5, \zeta_{f}(t)$ is an allowed Lefschetz zeta function for $N_{g}$. Hence $\operatorname{MPer}_{L}(f)=\{1, g\}$.

We consider $p(t)=c_{2 g}(t)$. By properties (p1) and (p2): $p(t)=\left(1+t^{g}\right)(1+t)^{-1}$. So by Proposition $5, \zeta_{h}(t)=p(t)(1-t)^{-1}$ is an allowed Lefschetz zeta function on $N_{g}$. Therefore

$$
\zeta_{h}(t)=\frac{p(t)}{1-t}=\frac{c_{2 g}(t)}{c_{1}(t)}=\frac{1+t^{g}}{(1+t)(1-t)}=\frac{1+t^{g}}{1-t^{2}}
$$

its $\operatorname{MPer}_{L}(h)$ is $\{1, g\} \cap\{2, g\}=\{g\}$.

Proof of Theorem 9(b). By property (p4) of the cyclotomic polynomials $c_{p^{\alpha}}(t)=(1-$ $\left.t^{p^{\alpha}}\right)\left(1-t^{p^{\alpha-1}}\right)^{-1}$. Let $p(t)=c_{p^{\alpha}}(t) c_{1}(t)$, whose degree is $g$. By Proposition 5, $\zeta_{f}(t)=p(t)(1-t)^{-1}$ is an allowed Lefschetz zeta function for $N_{g}$. Then $\operatorname{MPer}_{L}(f)=$ $\left\{1, p^{\alpha-1}, p^{\alpha}\right\}$

Proof of Theorem 9(c). Let $p(t)=c_{1}(t)^{l+1} c_{2}(t)^{l}$, where $2 l+1=g-1$, with $l \geq 0$. Since the degree of $p(t)$ is $g-1, \zeta_{f}(t)=p(t)(1-t)^{-1}$ is an allowed Lefschetz zeta function for $N_{g}$. So

$$
\zeta_{f}(t)=\frac{p(t)}{1-t}=c_{1}(t)^{l} c_{2}(t)^{l}=(1-t)^{l}(1+t)^{l}=\left(1-t^{2}\right)^{l} .
$$

Hence $\operatorname{MPer}_{L}(f)=\emptyset$.

Proof of Theorem 9(d). For $g=1$ the statement follows from Theorem 8. Assume $g>1$ odd. According to Proposition 5, the allowed Lefschetz zeta functions for $N_{g}$ are of the form $\zeta_{f}(t)=\frac{p(t)}{1-t}$, where $p(t)$ is a product of cyclotomic polynomials having degree $g-1>1$.

If $\operatorname{MPer}_{L}(f)=\emptyset$ then, from Proposition 7, $p(t)$ must satisfies:

(ii) If for $r>1$ odd and $m \geq 1$ the biggest positive integer such that $\left(1-\Delta t^{r}\right)^{m}$ is a factor of $p(t)$, then $\left(1+\Delta t^{r}\right)^{m}$ is also a factor of $p(t)$ having the biggest exponent, where $\Delta= \pm 1$.

(iii) If $m \geq 1$ is the biggest positive integer such that $(1-t)^{m}$ is a factor of $p(t)$, then $(1+t)^{m-1}$ is also a factor of $p(t)$ with the biggest exponent.

Note that under these two assumptions the Lefschetz zeta function $\zeta_{f}(t)$ is either 1, or it can be written as the product of factors of the form $\left(1+\Delta t^{2 r}\right)^{m}$. In both cases the degree of the polynomial $p(t)$ is odd.

Since if $g$ is odd, the degree $g-1$ of $p(t)$ is even, we have that $\operatorname{MPer}_{L}(f) \neq \emptyset$.

Proof of Theorem 9(e). According to Proposition 5 an allowed Lefschetz Zeta function is of the form $p(t)(1-t)^{-1}$ where $p(t)$ is a product of cyclotomic polynomials of total degree $g-1$. If $g>2$ then we consider $p(t)=\left(c_{1}(t)\right)^{g-1}$ then $\zeta_{f}(t)=(1-t)^{g-2}$, so its $\operatorname{MPer}_{L}(f)$ is $\{1\}$. If $g=2$, let $p(t)=c_{2}(t)$, so $\zeta_{f}(t)=(1+t)(1-t)^{-1}$; hence $\operatorname{MPer}_{L}(f)=\{1\}$. For $g=1$ the only allowed Lefschetz zeta function is $(1-t)^{-1}$, so its $\operatorname{MPer}_{L}(f)$ is $\{1\}$. 
Proof of Theorem $9(f)$. If $g$ is prime then $p=g$ and by the statement (a) of Theorem 9 the result holds.

If $g$ is not prime. We consider $p(t)=c_{2 p}(t)\left(c_{1}(t)\right)^{l}\left(c_{2}(t)\right)^{l}$, with $l \geq 0$, so that its total degree is $g-1$, i.e. $p-1+2 l=g-1$, see the properties (p1) and (p2) of the cyclotomic polynomials. According to Proposition $5, \zeta_{f}(t)=p(t)(1-t)^{-1}$ is an allowed Lefschetz zeta function for $N_{g}$. So

$\zeta_{f}(t)=\frac{p(t)}{1-t}=\frac{c_{2 p}(t)\left(c_{1}(t)\right)^{l}\left(c_{2}(t)\right)^{l}}{1-t}=\frac{\left(1+t^{p}\right)(1-t)^{l}(1+t)^{l}}{(1-t)(1+t)}=\left(1+t^{p}\right)\left(1-t^{2}\right)^{l-1}$.

Hence $\operatorname{MPer}_{L}(f)=\{p\}$.

Proof of Theorem 9(g.1). If $k$ is odd, i.e. $k=2 l+1$, let

$$
\begin{aligned}
p(t) & =c_{2 p_{1}}(t) c_{p_{2}}(t) c_{2 p_{3}}(t) \cdots c_{p_{k-1}}(t) c_{2 p_{k}}(t) \\
& =\left(\frac{1+t^{p_{1}}}{1+t}\right)\left(\frac{1-t^{p_{2}}}{1-t}\right) \cdots\left(\frac{1-t^{p_{k-1}}}{1-t}\right)\left(\frac{1+t^{p_{k}}}{1+t}\right) \\
& =\left(\prod_{i=1}^{k}\left(1+(-1)^{i+1} t^{p_{i}}\right)\right)\left(1-t^{2}\right)^{-l}(1+t)^{-1} .
\end{aligned}
$$

Then

$$
\zeta_{f}(t)=\frac{p(t)}{1-t}=\left(\prod_{i=1}^{k}\left(1+(-1)^{i+1} t^{p_{i}}\right)\right)\left(1-t^{2}\right)^{-l-1},
$$

so $\operatorname{MPer}_{L}(f)=\left\{p_{1}, \ldots, p_{k}\right\}$, and

$$
g=\left(\sum_{i=1}^{k} \varphi\left(p_{i}\right)\right)+1=\left(\sum_{i=1}^{k}\left(p_{i}-1\right)\right)+1=\left(\sum_{i=1}^{k} p_{i}\right)-(k-1),
$$

where $\varphi$ is the Euler function.

If $k$ is even, $k=2 l$, let

$$
\begin{aligned}
p(t) & =c_{p_{1}}(t) c_{2 p_{2}}(t) c_{p_{3}}(t) \cdots c_{p_{k-1}}(t) c_{2 p_{k}}(t) c_{1}(t) \\
& =\left(\prod_{i=1}^{k}\left(1+(-1)^{i} t^{p_{i}}\right)\right)\left(1-t^{2}\right)^{-l}(1-t) .
\end{aligned}
$$

So $\operatorname{MPer}_{L}(f)=\left\{p_{1}, \ldots, p_{k}\right\}$, and

$$
g=\left(\sum_{i=1}^{k} \varphi\left(p_{i}\right)\right)+2=\left(\sum_{i=1}^{k} p_{i}\right)-(k-2) .
$$

Proof of Theorem 9(g.2). Take

$$
\begin{aligned}
& q_{i}(t)=c_{2 p_{i}^{\alpha_{i}}}(t) c_{2 p_{i}^{\alpha_{i}-1}}(t) \cdots c_{2 p_{i}}(t) \\
& =\left(\frac{1+t^{p_{i}^{\alpha_{i}}}}{1+t^{p_{i}^{\alpha_{i}-1}}}\right)\left(\frac{1+t^{p_{i}^{\alpha_{i}-1}}}{1+t^{p_{i}^{\alpha_{i}-2}}}\right) \cdots\left(\frac{1+t^{p_{i}}}{1+t}\right) \\
& =\frac{1+t^{p_{i}^{\alpha_{i}}}}{1+t},
\end{aligned}
$$


and $p(t)=q_{1}(t) \cdots q_{k}(t)\left(c_{2}(t)\right)^{k} c_{1}(t)$. So

$$
\zeta_{f}(t)=\frac{p(t)}{(1-t)}=q_{1}(t) \cdots q_{k}(t)\left(c_{2}(t)\right)^{k}=\prod_{i=1}^{k}\left(1+t^{p_{i}^{\alpha_{i}}}\right) .
$$

Hence $\operatorname{MPer}_{L}(f)=\left\{p_{1}^{\alpha_{1}}, \ldots, p_{k}^{\alpha_{k}}\right\}$.

Proof of Theorem 9(g.3). Working similarly to the previous proof we take

$$
q_{i}(t)=c_{2 p^{\alpha_{i}}}(t) c_{2 p^{\alpha_{i}-1}}(t) \cdots c_{2 p}(t)=\frac{1+t^{p^{\alpha_{i}}}}{1+t}
$$

and $p(t)=q_{1}(t) \cdots q_{k}(t)\left(c_{2}(t)\right)^{k} c_{1}(t)$. So $\operatorname{MPer}_{L}(f)=\left\{p^{\alpha_{1}}, \ldots, p^{\alpha_{k}}\right\}$.

Proof of Theorem 9(g.4). Combining the proofs of statements (g.2) and (g.3) it follows easily the proof of this statement.

In view of the previous results we have the following open question.

Open question. Can be any finite set of odd positive integers the minimal set of Lefschetz periods for a $C^{1}$ Morse-Smale diffeomorphism on some non-orientable compact surface without boundary with a convenient genus?

We believe that the answer to this open question is positive. In [17] this open question was stated as a conjecture for the $C^{1}$ Morse-Smale diffeomorphisms on orientable compact surface without boundary.

\section{REFERENCES}

[1] Ll. Alsedà, J. Llibre and M. Misiurewicz, Combinatorial Dynamics and Entropy in dimension one (Second Edition), Advanced Series in Nonlinear Dynamics, vol 5, World Scientific, Singapore 2000.

[2] I.K. BABENKO AND S.A. BAGATYI, The behavior of the index of periodic points under iterations of a mapping, Math. USSR Izvestiya 38 (1992), 1-26.

[3] S. Batterson, The dynamics of Morse-Smale diffeomorphisms on the torus, Trans. Amer. Math. Soc. 256 (1979), 395-403.

[4] S. Batterson, Orientation reversing Morse-Smale diffeomorphisms on the torus. Trans. Amer. Math. Soc. 264 (1981), 29-37.

[5] S. Batterson, M. Handel and C. Narasimhan, Orientation reversing Morse-Smale diffeomorphisms of $\mathbb{S}^{2}$. Invent. Math. 64 (1981), 345-356.

[6] R.F. Brown, The Lefschetz fixed point theorem, Scott, Foresman and Company, Glenview, IL, 1971.

[7] J. Casasayas, J. Llibre and A. Nunes, Periods and Lefschetz zeta functions, Pacific Journal of Mathematics 165 (1994), 51-66.

[8] N. Fagella And J. Llibre, Periodic points of holomorphic maps via Lefschetz numbers, Trans. Amer. Math. Soc. 352 (2000), 4711-4730.

[9] J. Franks, Some smooth maps with infinitely many hyperbolic points, Trans. Amer. Math. Soc. 226 (1977), 175-179,

[10] J. Franks, Homology and dynamical systems, CBSM Regional Conf. Ser. in Math. 49, Amer. Math. Soc., Providence, R.I. 1982.

[11] J. Franks and C. Narasimhan, The periodic behaviour of Morse-Smale diffeomorphisms, Invent. Math. 48 (1978), 279-292.

[12] J.L. García Guirao and J. Llibre, Periods for the Morse-Smale diffeomorphisms on $\mathbb{S}^{2}$, Colloquium Mathematicum 200 (2007), 477-483.

[13] J.L. García Guirao and J. Llibre, Minimal Lefschetz sets of periods of Morse-Smale diffeomorphisms on the $n$-dimensional torus, J. of Difference Equations and Applications 16 (2010), 689-703.

[14] J.L. García Guirao and J. Llibre, The set of periods for the Morse-Smale diffeomorphisms on $\mathbb{T}^{2}$, to appear in Dynamical Systems: An International J.. 
[15] J. Guaschi and J. Llibre, Orders and periods of finite order homology surface maps, Houston J. of Math. 23 (1997), 449-483.

[16] A. Gulllamon, X. Jarque, J. Llibre, J. Ortega, J. Torregosa, Periods for Transversal Maps via Lefschetz Numbers for Periodic Points. Trans. Amer. Math. Soc.347 (1995), 47794806.

[17] B. Iskra and V.F. Sirvent, Cyclotomic Polynomials and Minimal sets of Lefschetz Periods. Pre-print.

[18] S. LAng, Algebra, Addison-Wesley, 1971.

[19] T.Y. Li and J. Yorke, Period three implies chaos, Amer. Math. Monthly 82 (1975), 985-992.

[20] J. Llibre, Lefschetz numbers for periodic points, Contemporary Math. 152 Amer. Math. Soc., Providence, RI, (1993), 215-227.

[21] J. Llibre and V.F. Sirvent, Minimal sets of periods for Morse-Smale diffeomorphisms on orientable compact surfaces, Houston J. of Math. 35 (2009), 835-855.

[22] J. Llibre and V.F. Sirvent, Erratum: Minimal sets of periods for Morse-Smale diffeomorphisms on orientable compact surfaces, Houston J. of Math. 36 (2010), 335-336.

[23] T. Matsuoka, The number of periodic points of smooth maps, Erg. Th. ES Dyn. Sys. 9 (1989), $153-163$.

[24] C. Narasimhan, The periodic behaviour of Morse-Smale diffeomorphisms on compact surfaces, Trans. Amer. Soc. 248 (1979), 145-169.

[25] J. Palis and W. De Melo, Geometric Theory of Dynamical Systems, An Introduction, Springer Verlag, New York, 1982.

[26] J. Palis and S. Smale, Structural stability theorems, Proc. Sympos. Pure Math. 14, Amer. Math. Soc., Providence, R.I., 1970, 223-231.

[27] C. Robinson, Dynamical Systems: Stability, Symbolic Dynamics and Chaos, Second Edition, CRC Press, Boca Raton, 1999.

[28] M. Shub, Morse-Smale diffeomorphisms are unipotent on homology, Dynamical systems (Proc. Sympos., Univ. Bahia, Salvador, 1971), Academic Press, New York, 1973.

[29] M. Shub And D. Sullivan, Homology theory and dynamical systems, Topology 14 (1975), $109-132$.

[30] S. Smale, Differentiable dynamical systems, Bull. Amer. Math. Soc. 73 (1967), 747-817.

1 Departament de Matemàtiques. Universitat Autònoma de Barcelona, Bellaterra, 08193 Barcelona, Catalunya, Spain

E-mail address: jllibre@mat.uab.cat

2 Departamento de Matemáticas, Universidad Simón Bolívar, Apartado 89000, CaraCAS 1086-A, VenEzuela

E-mail address: vsirvent@usb.ve 